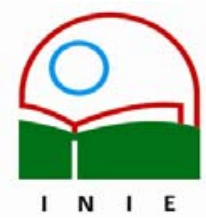

Actualidades Investigativas en Educación

Revista Electrónica publicada por el

Instituto de Investigación en Educación

Universidad de Costa Rica

ISSN 1409-4703

http://revista.inie.ucr.ac.cr

COSTA RICA

\title{
EL SOFTWARE MATEMÁTICO COMO HERRAMIENTA PARA EL DESARROLLO DE HABILIDADES DEL PENSAMIENTO $Y$ MEJORAMIENTO DEL APRENDIZAJE DE LAS MATEMÁTICAS
}

THE MATHEMATIC SOFTWARE AS A TOOL FOR DEVELOPING THINKING SKILLS AND IMPROVING THE LEARNING OF MATHEMATICS

\author{
Volumen 7, Número 2 \\ Mayo-Agosto 2007 \\ pp. 1-34
}

Este número se publicó el 30 de agosto 2007

\author{
Marisol Cuicas Ávila \\ Edie Debel Chourio \\ Luisa Casadei Carniel \\ Zulma Alvarez Vargas
}

La revista está indexada en los directorios:

LATINDEX, REDALYC, IRESIE, LASE, DIALNET, DOAJ, E-REVIST@S,

La revista está incluida en los sitios:

REDIE, RINACE, OEI, MAESTROTECA, HUASCARAN 


\title{
EL SOFTWARE MATEMÁTICO COMO HERRAMIENTA PARA EL DESARROLLO DE HABILIDADES DEL PENSAMIENTO Y MEJORAMIENTO DEL APRENDIZAJE DE LAS MATEMÁTICAS \\ THE MATHEMATIC SOFTWARE AS A TOOL FOR DEVELOPING THINKING SKILLS AND IMPROVING THE LEARNING OF MATHEMATICS
}

\author{
Marisol Cuicas Avila ${ }^{1}$ \\ Edie Debel Chourio ${ }^{2}$ \\ Luisa Casadei Carniel ${ }^{3}$ \\ Zulma Alvarez Vargas ${ }^{4}$
}

\begin{abstract}
Resumen: La investigación tuvo como propósito el desarrollo de habilidades del pensamiento y el mejoramiento del aprendizaje en alumnos y alumnas de la asignatura Matemática II, del Decanato de Ingeniería Civil de la Universidad Centroccidental "Lisandro Alvarado" (UCLA), mediante el empleo de estrategias instruccionales basadas en el uso del software matemático. La investigación, de diseño cuasi-experimental, usó para el contraste de hipótesis la prueba t para muestras relacionadas. Además se aplicaron dos pruebas, tres asignaciones, una lista de cotejo y una entrevista semiestructurada. Los datos recogidos se analizaron utilizando estadística descriptiva. Los hallazgos del estudio fueron: (a) los conocimientos de los discentes mejoraron; y (b) estos pusieron en práctica sus procedimientos (sus habilidades cognoscitivas y metacognitivas). Por lo tanto, el estudio aportó evidencias para usar el software matemático bajo una metodología constructivista.
\end{abstract}

Palabras claves: ESTRATEGIAS DE APRENDIZAJE, TECNOLOGÍA EDUCATIVA, HERRAMIENTAS COGNITIVAS, MATEMÁTICAS/ VENEZUELA/

\begin{abstract}
The purpose of this research project was to develop thinking skills and improve learning abilities of university students attending Math II, at the School of Civil Engineering at the University Centroccidental "Lisandro Alvarado" (UCLA), by utilizing instructional strategies, based on the use of mathematical software. The quasi experimental design of the study performed, to contrast the hypothesis, utilized test $t$ for related samples. Additionally, the following instruments were applied: two exams, three assignments, a checking list and a semi structured interview. The information collected was analyzed using descriptive statistics. The research findings were: (a) the students' knowledge improved; and (b) the students put into practice their procedures, that is to say, their cognitive and metacognitive skills. Hence, the study contributed to evidence the utilization of the mathematical software using a constructivist instructional methodology.
\end{abstract}

Key Words: LEARNING STRATEGIES, EDUCATIONAL TECHNOLOGY, TOOL COGNITIVE/ MATHEMATICS/ VENEZUELA

\footnotetext{
${ }^{1}$ Investigadora de la Universidad Centroccidental "Lisandro Alvarado". Correo electrónico: mcuicas@ucla.edu.ve

2 Investigador de la Universidad Nacional Experimental "Francisco de Miranda". Correo electrónico: ediedebel@yahoo.com

3 Investigadora de la Universidad Centroccidental "Lisandro Alvarado". Correo electrónico: luisacasadei@ucla.edu.ve

${ }^{4}$ Investigadora de la Universidad Centroccidental "Lisandro Alvarado". Correo electrónico: zulmaa@ucla.edu.ve
}

Artículo recibido: 30 de mayo, 2007

Aprobado: 28 de agosto, 2007 


\section{Introducción}

Para un ingeniero o ingeniera, la matemática constituye una herramienta para resolver problemas de ingeniería, sin olvidar que esta sirve: (a) como herramienta de cálculo; (b) para lograr el desarrollo del pensamiento lógico, algorítmico y heurístico y (c) como lenguaje universal capaz de contribuir al conocimiento y desarrollo de otras disciplinas propias de su perfil profesional. Así, la matemática es una herramienta de trabajo y, además es una disciplina fundamental en la formación de un profesional en ingeniería. Por ello, se debe lograr que su enseñanza sea eficiente, para que el alumno y la alumna adquieran los aprendizajes que los conduzcan a un mejor desenvolvimiento académico y profesional.

De acuerdo a Ángel y Bautista (2001), se debe convertir al alumnado en profesionales creativos, con capacidad de raciocinio, sentido crítico, intuición y recursos matemáticos que les puedan ser útiles. Por lo tanto, el profesorado está obligado a buscar herramientas que permitan la utilización de tecnologías para crear y proporcionar un ambiente de trabajo dinámico e interactivo. Herramientas, que permitan cambiar las metodologías de trabajo para la enseñanza y el aprendizaje, desarrollar habilidades del pensamiento propias del área de matemática y mejorar el aprendizaje en los alumnos y las alumnas.

La enseñanza de la matemática, comienza a caracterizarse por el uso de software como una herramienta didáctica. Estas herramientas adolecen de explicaciones teóricas y de insuficientes estrategias pedagógicas (Fernández, Izquierdo y Lima, 2000). Sin embargo, para Ángel y Bautista (2001), Balderas (2002), Dávila et al. (1998), Galdo y Cociña (1998), la evolución que ha experimentado el software, nos ofrece nuevas formas de enseñar, aprender y hacer matemática, brindando amplias posibilidades didácticas. Así mismo, destacan el potencial de esta tecnología tanto para lograr la interacción del alumnado con situaciones de aprendizaje que lo conduzcan a construir conocimientos, como para tener una visión más amplia del contenido matemático (Guedez, 2005). De allí, el interés de investigar sobre la aplicación de estrategias donde se usó el software Maple como herramienta cognitiva, con el objeto de contribuir al desarrollo de habilidades del pensamiento. Específicamente, se investigó sobre cómo innovar el proceso de aprendizaje en el tema de la integral definida incorporando el software Maple al trabajo intelectual del discente con el objeto de mejorar la comprensión y el aprendizaje de la matemática en estudiantes de Ingeniería Civil, de la Universidad Centroccidental "Lisandro Alvarado" (UCLA). 


\section{Planteamiento del problema}

En la enseñanza universitaria del Decanato de Ingeniería Civil (DIC) de la UCLA, el uso de tecnologías y metodologías instruccionales adaptadas a éstas ha sido lento. En el área de matemática, no existen propuestas concretas enmarcadas en una política, solo experiencias aisladas. Además, los métodos usados se centran en un proceso de información y preparación técnica, olvidando que la enseñanza es un proceso de formación, de acceso al pensamiento crítico y a la construcción del saber. Esta situación impide una mayor efectividad del DIC. En tal sentido, se hace necesario organizar una enseñanza que impulse el desarrollo de estas capacidades.

Según Segura y Chacón (1996), la enseñanza tradicional no proporciona al alumno o alumna herramientas para indagar, analizar y discernir la información. Para estas autoras, "los conocimientos impartidos son más bien automatizados, memorísticos y no fomentan el desarrollo de la iniciativa, la creatividad, ni la capacidad para comunicarse por distintas vías" (p. 29). Así mismo, Coll, Pozo, Sarabia y Valls (1992) afirmaron que la importancia del aprendizaje está en que el alumnado construya significados y atribuya sentido a lo que aprende; pues para un ingeniero o ingeniera, no basta adquirir conocimiento matemático, es determinante comprenderlo y aplicarlo.

Atendiendo a estos planteamientos, en el proceso de enseñanza en el área de matemática del DIC, se hizo necesario el empleo de métodos adecuados en la dirección del aprendizaje, que no solo se centren en trasmisión de contenidos, sino en el desarrollo de procesos del pensamiento propios de la matemática. Pues es claro que los procesos eficaces del pensamiento que no se vuelven obsoletos con rapidez, constituyen lo más valioso que les podemos proporcionar a las personas jóvenes (De Guzmán, 1993).

Para Gavilán, Ariza, Sánchez y Barroso (1999), con los avances tecnológicos existen muchos programas matemáticos como: Derive, Maple, Mathematica, Mathlab, los cuales proporcionan medios para la enseñanza de la matemática; sin embargo el/la docente debe saber aprovecharlos para generar situaciones que permitan al alumnado construir un conocimiento más significativo (Ángel y Bautista, 2001). La idea es que el discente use la tecnología computacional como herramienta cognitiva; es decir, como compañera intelectual del aprendiz para facilitar el pensamiento de alto nivel (Jonassen, 1996).

Sobre la base de lo expuesto para resolver este problema la investigación tuvo como propósito aplicar estrategias, en los estudiantes y las estudiantes de la asignatura Matemática II del DIC, donde se usó el software Maple como herramienta cognitiva, con el objeto de contribuir a desarrollar habilidades del pensamiento. Todo esto, con el fin de 
mejorar la comprensión y el aprendizaje de la población de estudiantes en el tema de la integral definida, lo que afecta el rendimiento académico en matemática. Para ello, se partió del supuesto de que las tecnologías sirven de apoyo al aprendizaje y no pretenden la instrucción del alumno o alumna, sino "servir de herramientas de construcción del conocimiento, para que los estudiantes aprendan con ellas, no de ellas" (Jonassen, Carr y Ping, 1998, p. 1). En tal sentido, el software se usó con fines didácticos para facilitar estrategias de enseñanza-aprendizaje relacionadas con el tema de la integral definida.

\subsection{Interrogantes de la investigación}

Esta investigación pretendió resolver un problema del DIC como fue mejorar la comprensión y el aprendizaje del tema de la integral definida en las personas cursantes de la asignatura Matemática II del DIC. En tal sentido, los investigadores e investigadoras se plantearon la siguiente interrogante: ¿cuál es la relación entre el uso de estrategias instruccionales basadas en el software matemático, orientadas al desarrollo de habilidades del pensamiento y a la obtención de mejoras en el aprendizaje en la asignatura Matemática II? Esta pregunta tuvo implícitos dos aspectos, el desarrollo de habilidades del pensamiento y la obtención de mejoras en el aprendizaje.

Para Sánchez (2002), el desarrollo de habilidades del pensamiento requiere el uso de conocimientos, procedimientos y actitudes. Las mejoras en estos aspectos conducirán a mejorar el aprendizaje. Las habilidades del pensamiento son capacidades que permiten el dominio de la realización de una actividad (Sarmiento, 1999). El desarrollo de las habilidades del pensamiento significa que la capacidad de pensar puede nutrirse y cultivarse en las personas (Sánchez, 2002). Para estas autoras, las mencionadas habilidades pueden ser desarrolladas y adquiridas por las personas a través de su formación, práctica, estudio, conocimientos y actitudes. No obstante, en está investigación solo se trataron aspectos relacionados con los conocimientos y procedimientos.

Los conocimientos correspondieron con el saber de la materia o área en la cual se piensa (Sánchez, 2002), y abarcan conocimientos conceptuales y conocimientos de procedimientos. Los conocimientos conceptuales se construyen a partir del aprendizaje de conceptos, principios y explicaciones; no tienen que ser aprendidos en forma literal, sino abstrayendo su significado esencial e identificándolo con las características definitorias o reglas que lo componen (Díaz y Hernández, 2002). Ellos incluyeron conocer sobre la materia: fuentes confiables de datos, heurísticas especiales para manejar los datos, conceptos específicos, leyes, teoremas, conocimiento de representaciones gráficas, entre 
otros. Para Díaz y Hernández, los conocimientos de procedimientos son de tipo práctico y están basados en acciones ordenadas, dirigidas hacia la consecución de una meta determinada. Se refieren a la ejecución de procedimientos, estrategias, técnicas, métodos, etc. En tal sentido, ellos incluyeron: manejo de conceptos, símbolos o términos básicos; uso de algoritmos; manejo de técnicas, realización de representaciones gráficas, entre otros.

Los procedimientos tienen que ver con las operaciones que ejecuta la persona para aplicar el conocimiento. Según Sánchez (2002), estas operaciones son de tipo cognoscitivas y metacognitivas. Para Sánchez, las cognoscitivas comprenden las operaciones para generar o aplicar el conocimiento; incluyen una serie de procesos y estrategias que facilitan la toma de decisiones, la resolución de problemas y la conceptualización, así como los siguientes procesos: observar, conocer, relacionar, analizar, inferir, sintetizar y concluir. Las metacognitivas permiten dirigir y controlar la producción de significados, así como darle sentido al pensamiento a través de los procesos de planificación, formulación, ejecución, supervisión y evaluación. En tal sentido, los procedimientos tuvieron que ver con la interpretación de expresiones gráficas y/o simbólicas, análisis, síntesis, realización de inferencias, elaboración de conclusiones a partir de la información afín que se conoce; planificación de acciones, toma de decisiones, evaluación y comunicación de resultados, entre otros.

En relación con lo anterior, esta investigación trató de responder las siguientes preguntas: (a) ¿qué relación existe entre el uso de estrategias instruccionales basadas en el software matemático y la obtención de mejoras del conocimiento de la asignatura Matemática II en el/la estudiante?, y (b) ¿qué relación existe entre el uso de estrategias instruccionales basadas en el software matemático y el desarrollo de habilidades relacionadas con el uso de procedimientos en la población de estudiantes cursantes de la asignatura Matemática II?

\subsection{Hipótesis de la investigación}

Las hipótesis nulas verificadas en el estudio fueron las siguientes: (a) no existe relación entre el uso de estrategias instruccionales basadas en el software matemático y la obtención de mejoras del conocimiento de la asignatura Matemática II en el/la estudiante, y (b) no existe relación entre el uso de estrategias instruccionales basadas en el software matemático y el desarrollo de habilidades relacionadas con el uso de procedimientos en estudiantes cursantes de la asignatura Matemática II.

Las hipótesis alternativas fueron: (a) existe relación entre el uso de estrategias 
instruccionales basadas en el software matemático y la obtención de mejoras del conocimiento de la asignatura Matemática II en el/la estudiante, y (b) existe relación entre el uso de estrategias instruccionales basadas en el software matemático y el desarrollo de habilidades relacionadas con el uso de procedimientos en estudiantes cursantes de la asignatura Matemática II.

\section{Revisión de la literatura}

La teoría constructivista considera que el aprendizaje es siempre una construcción interior (Flórez, 2000). Para Díaz y Hernández (2002), un concepto central en la teoría constructivista es el aprendizaje significativo, definido por Ausubel como el proceso usado por el alumno y la alumna para aprender, "el cual relaciona la información nueva con la que posee, dándole un significado y favoreciendo su comprensión" (Sarmiento, 1999, p. 309). Ello implica considerar las ideas previas del alumnado y "reconocer el nivel de pensamiento lógico que posee el alumno para proporcionarle experiencias que promuevan sus habilidades del pensamiento" (Flórez, 2000, p. 246).

El constructivismo abre posibilidades para promover estrategias que, incorporando tecnología informática, favorezcan la creación de nuevas formas de aprendizaje centradas en el aprendiz (Dede, 2000). Pues el empleo de las tecnologías, conectándolas con experiencias significativas, pueden constituir herramientas cognitivas que el discente utiliza para estimular y desarrollar habilidades del pensamiento (Jonassen, Carr y Ping, 1998). Así, según estos autores aprender con el computador supone el efecto de la tecnología en el aprendiz que participa intelectualmente con dicha herramienta, la cual permite al alumno o alumna organizar las ideas con mayor soltura para actuar posteriormente con ellas apoyando su proceso de aprender (Esteban, 2002).

Las herramientas cognitivas son dispositivos usados para visualizar, organizar, automatizar o suplantar las técnicas del pensamiento (Jonassen, 1996). Es decir, la idea es que el alumnado use la tecnología como herramienta para (Esteban, 2002): (a) representar el problema, (b) promover sus conocimientos, (c) consolidar esquemas preexistentes mediante la automatización de ejercicios de un nivel inferior, y (d) reagrupar la información pertinente y necesaria al resolver un problema. Así, en esta modalidad de herramienta cognitiva la tecnología se hace cargo de las actividades trabajosas y rutinarias (calcular, graficar). Esto permite que el alumno y la alumna se centren en conceptos esenciales y ayuda el/al docente a evitar actividades que no aportan nada en forma directa a la tarea educativa pero que hace falta realizar (Squires y McDougall, 1997). 
Por lo expuesto, el uso del computador como herramienta mental se concentra en la calidad de la idea, ya que con este se pueden realizar manipulaciones (calcular, graficar, trasladar, ordenar) permitiendo generar y organizar las ideas más fácilmente, apoyando el proceso de aprender. Es evidente que bajo esta perspectiva "el profesor debe encarar un rol de gerenciador de saberes y desarrollador de habilidades que permitan a los alumnos utilizar el análisis crítico y reflexivo" (Cataldi, 2000, p. 16).

El uso de tecnologías en la enseñanza de la matemática permite en el alumnado el desarrollo de habilidades del pensamiento como: explorar, inferir, hacer conjeturas, justificar, argumentar y de esta forma construir su propio conocimiento (Fernández, Izquierdo y Lima, 2000). Para estos autores, estas habilidades pueden ser desarrolladas integrando al trabajo intelectual del alumno y de la alumna el software matemático. Además, dicha relación puede generar variadas "experiencias y aplicaciones orientadas a producir, calcular, graficar, modelar, explorar, visualizar, clasificar, comparar, aplicar, informar, simular o aplicaciones en que se integra la matemática a otras disciplinas" (Oteiza y Silva, 2001, p. 2). En tal sentido, con el uso del software la atención se enfoca en facilitar que el discente aprenda a procesar la información de la materia, así como, en la transferencia y generalización de los aprendizajes a otros aspectos académicos o no. Para Martín (2001) y Sánchez (2002), estos aspectos son primarios para el desarrollo de las habilidades del pensamiento de orden superior.

Por otra parte, Sánchez (2002), agregó que en el desarrollo de habilidades del pensamiento, es importante conocer de una materia fuentes de datos, heurísticas especiales para manejar datos, así como conceptos específicos para generar, organizar y darle sentido a la información. Sin embargo, para Sánchez este conocimiento no es sustituto de la habilidad para manejar las operaciones del pensamiento. Ambos aspectos, el conocimiento de la materia y la habilidad para manejar las operaciones del pensamiento son esenciales para lograr un pensamiento productivo.

En el desarrollo de habilidades del pensamiento, el papel del educador es esencial ya que debe ayudar al alumnado a modificar sus estructuras cognoscitivas (Díaz y Hernández, 2002). Para ello, el/la docente debe adquirir una metodología que suponga un cambio de actitud hacia la enseñanza, donde los estudiantes y las estudiantes trabajen, reflexionen y elaboren sus propios procesos del pensamiento (Martín, 2001). En este sentido, Díaz y Hernández (2002) y Sarmiento (1999), indicaron que este tipo de aprendizaje se fomenta: (a) enseñando habilidades del pensamiento como estrategias a las que se puede tener acceso, (b) facilitando que el discente sea consciente de sus objetivos y decisiones estratégicas y (c) 
enseñando tanto el dominio de los procesos implicados en una habilidad como el conocimiento de cuándo y porqué usar esa habilidad. A este respecto, para Hernández y Sancho (1993) la finalidad de aprender estrategias de aprendizaje es facilitar la automatización de una serie de pautas que pueden ser aplicadas a otras situaciones, cuando las circunstancias de resolución de problemas, planificación de un trabajo, organización de la información lo requieran. Así, lo que se busca es facilitar estrategias que mejoren las habilidades del pensamiento, partiendo de lo que el/la estudiante es capaz de hacer y avanzar gradualmente hacia habilidades de orden superior.

Por otra parte, las herramientas informáticas permiten introducir una metodología de trabajo más constructivista en las clases de matemática, promoviendo una participación activa y creativa del aprendiz (Ángel y Bautista, 2001; Guedez, 2005; Oteiza y Silva, 2001). Así mismo, dichos autores destacan que con el uso adecuado de estas herramientas el alumno y la alumna, asesorados por el profesor, pueden realizar actividades que les permitan conjeturar, explorar, experimentar y extraer conclusiones. Dichos procesos, les fomentan en el discente la toma de conciencia de la factibilidad de sus ideas, haciendo su aprendizaje más comprensivo que memorístico (Garza y Leventhal, 2004). Sin embargo, "esto no implica dejar a un lado la memoria, sino hacer uso adecuado de ella" (Garza y Leventhal, 2004, p. 29).

\subsection{Software matemático y estrategias de enseñanza-aprendizaje}

Las herramientas informáticas abarcan sistemas de simulación y modelado, software matemático, sistemas multimedia, entre otros. Los beneficios que se obtengan de su uso en la labor docente, estarán en función de la capacidad que se tenga de su manejo y adecuación (Meza y Cantarell, 2002).

Con el uso adecuado del software matemático, el/la docente debe convertirse en un facilitador y diseñador de situaciones de aprendizaje para desarrollar en el alumnado habilidades de autoaprendizaje (Meza y Cantarell, 2002). Su uso permite la interacción entre el/la docente y el discente, "generando una dinámica enriquecedora para ambos, en la que el centro del proceso es el estudiante, el cual se hace responsable por la calidad del aprendizaje" (Ríos, 1998, p. 4). Adicionalmente, para Ángel y Bautista (2001) con el empleo del software matemático, el/la docente debe adaptar su metodología a esta herramienta e integrar los conocimientos teóricos y prácticos, así como diseñar aplicaciones y problemas orientados al uso del software. Sin olvidar que diseñar este tipo de actividades requiere buen conocimiento del software, coherencia didáctica respecto a lo que se le propone al alumnado 
y ofrecer a este último una guía de cómo, cuándo y para qué utilizar esta herramienta (Ángel y Bautista, 2001).

Por otra parte, software como Derive, Mathematica, Maple, Mathlab, entre otros, presentan muchas posibilidades para introducir al alumno y alumna en una actividad matemática de orden superior (Ángel y Bautista, 2001; Anido, López y Rubio, 2006; Galdo y Cociña, 1998; Oteiza y Miranda, 2001; Queralt, 2000). Según estos autores, estas herramientas hacen viable el diseño de estrategias para la práctica del contenido matemático. En este sentido, Ángel y Bautista (2001), Balderas (2002), Galdo y Cociña (1998), Orellana (1999), y Queralt (2000) argumentaron que entre las posibilidades del software están: (a) favorece los procesos inductivos y visualización de conceptos; (b) permite comparar, verificar, conjeturar y refutar hipótesis; (c) posibilita tener un laboratorio de cálculo; (d) individualiza el proceso de enseñanza-aprendizaje; (e) sirve como elemento de motivación y como instrumento generador de problemas matemáticos y (f) facilita la comprensión y aprendizaje de los contenidos programáticos.

Cabe destacar, que el uso de tecnología no es la solución de todos los problemas educativos (Dede, 2000; Guedez, 2005), pues el valor de usar computadoras estará en función de lo que diseñen los educadores, pero sobre todo de lo que haga el discente con ellas (Meza y Cantarell, 2002). En tal sentido, la tarea del docente es planificar, desarrollar y evaluar procesos de enseñanza-aprendizaje, donde el software representa el papel de herramienta cognitiva. No obstante, se debe cuidar que el software no se constituya el objeto de estudio, descuidando el aprendizaje de temas esenciales que se deben lograr con el uso de estos recursos (Meza y Cantarell, 2002).

Ríos (1998), expuso una experiencia donde usó el Mathlab y Anugraph para que el alumnado pudiera "experimentar con los objetos matemáticos y sus propiedades, hacer conjeturas y descubrir por sí mismo resultados importantes" (Ríos, 1998, p. 2). Esto permitió reforzar la comprensión intuitiva de los conceptos y la creatividad, ayudando a eliminar el excesivo énfasis en aspectos calculistas, rescatando la idea de que el aprendiz es el responsable de su aprendizaje. Bajo este enfoque el alumno, la alumna y la tecnología actúan como socios, pues aquellos planifican, interpretan, deciden, descubren y el software calcula, grafica, almacena, es decir efectúa actividades más rutinarias (Ríos, 1998). Así, el software es usado como una herramienta cognitiva pues asume aspectos de una tarea liberando espacio cognitivo que el alumnado puede emplear en pensamientos de nivel superior (Jonassen, Carr y Ping, 1998). En tal sentido, el computador les permite aplicar eficientemente sus esfuerzos y poner en marcha mecanismos más complejos del 
pensamiento (Marqués, 2001). Por lo expuesto, se trata de que el discente aprenda con la tecnología y no de ella (Jonassen, Carr y Ping, 1998).

Galdo y Cociña (1998) reportaron otra experiencia con el software Mathematica. Para estas autoras, el uso del software "favorece los procesos inductivos y la visualización de conceptos complejos. Permite comparar, verificar o refutar hipótesis, cambiar postulados y someterlos a prueba y conjeturar, apoyándose en la construcción de modelos" (Galdo y Cociña, 1998, p. 667). Además, señalaron que el software sirve de apoyo y ayuda para que el aprendiz delibere; sin embargo, el/la docente debe impartir la teoría previamente, orientar y motivar, dejando al alumnado buscar la independencia en su tarea.

Dávila et al. (1998), realizaron una experiencia con el software Derive para que los alumnos y las alumnas entrelazaran los conceptos matemáticos con los contenidos de otras asignaturas. Según estos autores, el software les proporcionó datos, gráficos, resultados que debieron interpretar para dar la solución y explicaciones al problema. Además, apuntaron que al usar el software se debe: (a) diseñar situaciones de aprendizaje donde el alumno o alumna desconozca si los resultados obtenidos están bien de forma inmediata; (b) diseñar prácticas que los obliguen a trabajar, a pensar, estudiar, para dar solución a los problemas y (c) fomentar la labor tutorial del profesor.

Fallad (1999) realizó investigaciones con un software educativo orientado al aprendizaje centrado en el discente y al aprendizaje colaborativo. Con ayuda del software, Fallad pretendió generar en la población estudiantil una formación de esquemas para la resolución de problemas matemáticos, permitiendo que el discente tome conciencia del proceso utilizado para trabajar. Aspecto importante para el desarrollo de las habilidades del pensamiento (Díaz \& Hernández, 2002; Sarmiento, 1999). Entre los resultados obtenidos por Fallad, para el discente el uso de un software: (a) conlleva un impacto notable en el aprendizaje de la matemática, reflejado posteriormente en su desempeño profesional; (b) le facilita el aprendizaje, pues le proporciona herramientas para resolver problemas, incorporar estrategias de aprendizaje colectivo y el trabajo en equipo; y (c) le permite realizar analogías o extrapolaciones a otros problemas.

Así mismo, Fernández, Izquierdo y Lima (2000), realizaron experiencias donde combinaron el empleo de software como Derive, Cabri-Geometry, Modelus, Statistica, Mathematica, Pramatic, Estadis y Calculus. Como conclusiones se destacaron: (a) utilizar el software ayuda en la formación de conceptos, ejercitación y resolución de problemas; (b) graficar, simplificar y realizar cálculos complicados de manera rápida aportó realismo a las aplicaciones; (c) caracterizar el software por su sencillez manipulativa, facilidades para la 
experimentación numérica y pocas exigencias del hardware, sin embargo carece de una adecuada estrategia pedagógica y requiere del conocimiento de los conceptos teóricos asociados y d) utilizar tecnología exigió del profesor una actividad mayor y constante creatividad, pues debió explorar el conocimiento previo del discente y proporcionarle un ambiente para que éste construya su conocimiento.

Ángel y Bautista (2001), también realizaron experiencias educativas utilizando en matemática software como Excel, Minitab, Mathcad y Mathematica. El objeto de la actividad fue aprender a usar los conceptos matemáticos en forma práctica, aumentar la capacidad de razonar de los estudiantes y las estudiantes, resolver problemas no rutinarios, comunicar y utilizar contextualmente las ideas matemáticas, entre otros. Ángel y Bautista argumentaron que diseñar actividades instruccionales e integrarlas en el programa de una asignatura requiere un buen conocimiento del software y una coherencia didáctica respecto a lo que se le propone al discente, teniendo en cuenta los contenidos que pretendemos reforzar con ellos. Para estos autores, es fundamental ofrecer al discente una guía de cómo, cuándo y para qué utilizar esta herramienta informática. Así, incluir el aprendizaje y el uso de software específico para las asignaturas de matemática a un nivel de enseñanza superior requiere replantearse, tanto la metodología como el propio currículum de la asignatura. Además señalaron, que el uso de software como herramienta posibilita la visualización de conceptos, pudiendo ser usada en diversos entornos profesionales o educativos.

Guedez (2005), presentó una investigación sobre el aprendizaje de funciones utilizando el software FunReal 1.0. Dicho software utilizado como herramienta de aprendizaje, permitió a la población de estudiantes ser más activos, participativos y autónomos en la adquisición de conocimientos, dinamizando con su uso el aula de clases y promoviendo en ellos/ellas un sentimiento de autonomía, en la adquisición de conocimientos.

Sobre la base de lo expuesto, cada día queda más claro que es importante el uso inteligente de los recursos informáticos, pues estos permiten ampliar las experiencias de aprendizaje (Dede, 2000). Para este autor no basta con emplear tecnología, "es necesario la capacitación de los docentes para afrontar estas situaciones y un proceso de aprendizaje de la comunidad toda para aceptar las diferencias" (Dede, 2000, p. 3). En tal sentido, se deben aprovechar estos recursos para innovar el currículum, la enseñanza y el aprendizaje. Sin olvidar que toda filosofía de trabajo requiere de tiempo y espacio tanto para comprenderla, adquirirla, desarrollarla y valorarla. 


\section{Metodología utilizada en el estudio}

\subsection{Tipo de investigación}

En la revisión de la literatura, se encontró suficiente evidencia para sustentar que la utilización adecuada del software matemático integrado al trabajo intelectual del alumno ayuda en el aprendizaje de la matemática. Sin embargo, en las experiencias registradas no se evidenciaron la metodología de investigación utilizada ni sugieren un tipo específico de investigación (cualitativa o cuantitativa). Además, la revisión reflejó la falta de experiencias en el área de matemática que puedan ser sistematizadas y preparadas para ser incorporadas a la práctica docente (Orellana, 1999; Oteiza y Silva, 2001; Ríos, 1998).

En tal sentido, se optó por realizar un estudio donde se recogieron datos cualitativos y cuantitativos. Esta decisión se debió a que autores como Fernández y Díaz (2003), Bavaresco (2001), y Buendía, Colás y Hernández (1998), argumentaron que el empleo de procedimientos cualitativos y cuantitativos en una investigación podría ayudar a corregir los sesgos propios de cada método. Así mismo, Sánchez (2002) señaló que en investigaciones relacionadas con el desarrollo de habilidades del pensamiento la combinación de ambas metodologías (cualitativa y cuantitativa) es adecuada, pues permite la vigorización mutua que propicia percepciones cualitativas y cuantitativas que ninguno de los dos podría lograr por separado.

Por otra parte, con la finalidad de determinar el resultado sobre el aprendizaje de la integral definida, valorado por el rendimiento académico, se seleccionó un diseño denominado diseño de un solo grupo con pretest y postest (Buendía, Colás y Hernández, 1998). No obstante, Fernández y Díaz (2003) manifestaron que los diseños de investigación son lineamientos generales por seguir, no son pasos en los que cualquier alteración puede acarrear graves consecuencias para el resultado final. Para estos autores, la pregunta de investigación limita los tipos de investigación que pueden emplearse, sin embargo no restringe la selección de una sola opción de diseño.

Sobre la base de lo expuesto, se optó por seleccionar un diseño cuasi-experimental, incluyendo en el procedimiento la recolección de datos cualitativos y cuantitativos. Los datos numéricos se agruparon y se tabularon para construir cuadros estadísticos. Los datos verbales se transformaron numéricamente, debiendo efectuar en ellos el proceso de agruparlos, categorizarlos, codificarlos, tabularlos, y finalmente fueron trasladados a cuadros (Bavaresco, 2001). Además, en este tipo de investigación (cuasi-experimental), se asumió que los individuos que participaron en ella conservaron "ciertas diferencias a pesar de recibir el mismo tratamiento de la variable independiente" (León y Montero, 2003, p. 328). 


\subsection{Variables de la investigación}

Las variables por considerar fueron (Tabla 1): a) estrategias instruccionales basadas en el software (variable independiente), y mejoras en los conocimientos y procedimientos (variables dependientes).

Tabla 1. Indicadores de las variables conocimientos y procedimientos

\begin{tabular}{|c|c|c|}
\hline $\begin{array}{l}\text { Conocimientos } \\
\text { Conceptuales }\end{array}$ & $\begin{array}{c}\text { Conocimientos de } \\
\text { Procedimientos }\end{array}$ & Procedimientos \\
\hline $\begin{array}{ll}\text { - } & \text { Conoce los } \\
\text { símbolos } \\
\text { utilizados } \\
\text { - Conoce los } \\
\text { algoritmos } \\
\text { - Conoce los } \\
\text { conceptos } \\
\text { - Conoce los } \\
\text { teoremas y leyes } \\
\text { - Conoce las } \\
\text { representaciones } \\
\text { gráficas } \\
\text { Conoce las } \\
\text { estrategias y } \\
\text { técnicas por } \\
\text { emplear }\end{array}$ & $\begin{array}{l}\text { - Maneja los símbolos } \\
\text { - } \text { Atilizados } \\
\text { de manera correcta } \\
\text { - Aplica los conceptos de } \\
\text { manera apropiada } \\
\text { - Aplica los teoremas y leyes } \\
\text { de forma lógica } \\
\text { - Realiza las representaciones } \\
\text { gráficas } \\
\text { - Maneja las técnicas utilizadas } \\
\text { - En la resolución de ejercicios } \\
\text { - emplica la técnica o estrategia } \\
\text { - Usa diversas estrategias o } \\
\text { - técnicas } \\
\text { - Presenta un trabajo en forma } \\
\text { lógica y ordenada }\end{array}$ & $\begin{array}{l}\text { - Usa contradicciones para aclarar } \\
\text { conceptos. } \\
\text { - Realiza procedimientos de ensayo y } \\
\text { error. } \\
\text { - } \text { Propone y explora conexiones entre } \\
\text { conceptos. } \\
\text { - Aplica los conceptos a situaciones } \\
\text { nuevas. } \\
\text { - Toma decisiones de acuerdo con } \\
\text { criterios válidos. } \\
\text { - Modela, explica y aplica una } \\
\text { estrategia. } \\
\text { - Evalúa la veracidad de los } \\
\text { resultados, con base en } \\
\text { procedimientos válidos. } \\
\text { - Genera nuevos procedimientos. } \\
\text { - Realiza inferencias } \\
\text { - } \text { Realiza generalizaciones. } \\
\text { y lógica. }\end{array}$ \\
\hline
\end{tabular}

\section{Participantes}

El estudio se realizó con 34 estudiantes inscritos en la asignatura Matemática II del programa de Ingeniería Civil del DIC. La sección donde se llevó a cabo el estudio fue seleccionada en forma aleatoria y estuvo compuesta por alumnos y alumnas repitientes. Las personas participantes poseían edades entre 19 a 23 años, eran de ambos sexos, bachilleres en ciencias y del mismo nivel socioeconómico. No tenían experiencia en el uso del software matemático Maple en cursos anteriores de matemática, pues este solo es utilizado en las asignaturas de semestres superiores. Además, la metodología incorporada a las clases de Matemática I y Matemática II, en la que fueron expuestos estos participantes por lo general es muy expositiva y tradicional.

Lo expuesto anteriormente permitió a los investigadores e investigadoras realizar ciertas comparaciones entre la nueva metodología empleada y la modalidad tradicional, logrando los alumnos y alumnas participantes establecer conclusiones sobre si el uso de 
esta herramienta informática les sirvió como apoyo para mejorar habilidades del pensamiento (relacionadas con los conocimientos y procedimientos) y su aprendizaje en matemática.

\subsection{Procedimiento}

1. Se realizaron prácticas con el grupo seleccionado, referidas al manejo básico del software Maple, sus usos y aplicaciones. Esto con el objetivo de ofrecer al discente una guía de cómo, cuándo y para qué usar esta herramienta, aspecto importante en el uso del software tanto para Ángel y Bautista (2001), como para Jonassen (1996).

2. Se administró una prueba exploratoria al grupo, con la que se determinó el nivel de conocimientos de los discentes en relación con el tema de la integral definida. Esta fue aplicada tanto al inicio como al final del experimento.

3. Se aplicó el tratamiento, el cual formó parte de las actividades de clase y estuvo a cargo de uno de los investigadores e investigadoras. Este consistió en el empleo de una metodología que incorporó situaciones de aprendizaje creadas de manera intencional, donde se usó el software Maple como herramienta cognitiva. Dichas actividades estuvieron relacionadas con asignaciones escritas, pruebas escritas y secuencias instruccionales diseñadas para tal fin. Las asignaciones tuvieron como objetivo comprometer al alumno y a la alumna en el aprendizaje de procedimientos (habilidades cognoscitivas y metacognitivas) mientras construían el conocimiento. Por lo tanto, utilizaron el software para representar lo que sabían, interpretando y organizando el conocimiento de tal forma que el software les sirviera de apoyo. Dichas asignaciones se realizaron en equipo (dos integrantes) y con asesoramiento docente. Así mismo, fueron entregadas para su evaluación en formato electrónico, utilizando para ello el software Maple. Su realización de las mismas se llevó a cabo una vez que se impartió el tema en la clase.

Se efectuaron evaluaciones formativas y sumativas. La evaluación formativa se llevó a cabo en horas de consulta y a través de los correos electrónicos. La evaluación sumativa se llevó a cabo mediante: (a) tres asignaciones, en las cuales se usó el software para facilitar el aprendizaje del concepto de la integral definida y su interpretación geométrica, de las reglas de Simpson y trapecial, y para la visualización de ejercicios relacionados con las integrales impropias, y (b) dos pruebas, realizadas de manera individual, siendo sus objetivos evaluar los conocimientos de los temas tratados.

Para verificar la primera hipótesis nula, se utilizaron las calificaciones obtenidas por los alumnos y las alumnas en las pruebas y las asignaciones. Las mismas establecieron el 
rendimiento y se les calculó la media y la desviación típica. La mencionada actividad estuvo acompañada por una lista de cotejo para registrar observaciones sobre algunas conductas del alumnado, observadas en las pruebas y asignaciones. Además, se aplicó una prueba $t$ de Student para muestras relacionadas con el objeto de comparar los resultados obtenidos en la aplicación de la prueba exploratoria, tanto al inicio como al final del experimento.

Para la segunda hipótesis, se empleó una lista de cotejo en las actividades inherentes a las horas de consultas. En esta actividad, los alumnos y las alumnas presentaban la asignación en formato electrónico para su discusión. Aquí, los estudiantes y las estudiantes tenían la oportunidad de preguntar para aclarar dudas, y el/la docente podía realizar observaciones y preguntas para verificar sus habilidades en los procedimientos 0 conocimientos. Por lo tanto, el trabajo en el computador constituyó el núcleo central de la actividad.

Para enriquecer las observaciones registradas en las listas de cotejo, se realizó una entrevista semi-estructurada al grupo. Dicha entrevista se efectuó una vez publicadas las calificaciones de la unidad. Con ella se logró obtener información complementaria sobre el empleo de procedimientos por parte de los alumnos y las alumnas. Cabe destacar, que se eligieron las respuestas más relevantes que pudieron arrojar información para el estudio y complementar la información obtenida a través de las listas de cotejo.

\subsection{Instrumentos}

1. Prueba exploratoria de conocimientos. Consistió en una prueba escrita sobre 20 puntos, de carácter formativo para las actividades académicas. Fue validada mediante un juicio de expertos. Su objetivo fue determinar el efecto del tratamiento al comparar los resultados de su aplicación antes y después del experimento (Hernández, Fernández y Baptista, 2003).

2. Dos pruebas escritas. La primera evaluó los logros de los alumnos y las alumnas en relación con el tema de la integral definida, y la segunda evaluó los logros correspondientes al tema de integrales impropias. Dichas pruebas fueron validadas mediante un juicio de expertos.

3. Tres asignaciones escritas. Validadas en contenido mediante un juicio de expertos.

4. Una lista de cotejo. Este instrumento estuvo estructurado en dos partes con alternativas de respuesta dicotómicas (sí y no), siendo elaborado en función de los indicadores de las variables conocimientos y procedimientos (Tabla 1).

5. Guión de la entrevista. Guía de interrogantes o aspectos donde los investigadores e 
investigadoras tuvieron la libertad de introducir preguntas adicionales para precisar conceptos y obtener mayor información sobre el tema deseado.

\section{Resultados}

\subsection{Primera hipótesis de investigación}

Para detectar mejoras en los conocimientos del alumnado se consideró el rendimiento académico, así como la prueba exploratoria. Para el contraste de la hipótesis, se aplicó la prueba $t$ de Student para muestras relacionadas con las calificaciones obtenidas en las pruebas exploratorias. Los resultados del cálculo de la prueba $t$ con un $\alpha=.05$ se muestran en la Tabla 2. No obstante, la media obtenida en la prueba exploratoria antes (9.96) y después del tratamiento (13) reporta diferencias, pues el grupo mejoró su promedio de notas. Además, el valor $p=0<.05$. Según Triola (2004), se rechaza la hipótesis nula y los resultados son significativos para el valor $\alpha=.05$. En tal sentido, las diferencias obtenidas apoyan la posibilidad de que existe relación entre el uso de estrategias basadas en el software matemático como herramienta cognoscitiva y la obtención de mejoras en los conocimientos de los discentes.

Tabla 2. Resultados de la prueba t al aplicar la prueba exploratoria

\begin{tabular}{lccccc}
\hline Prueba exploratoria & Media & Desviación típica & $t$ & $g l$ & Sig. bilateral \\
\hline Antes & 9.96 & 0.93 & & & \\
Después & 13.00 & 1.84 & & & \\
Antes-después & -3.04 & 1.88 & -9.44 & 33 & 0.00 \\
\hline
\end{tabular}

Por otra parte, la ausencia de un grupo control impidió conocer con exactitud si fue el tratamiento $u$ otros factores lo que produjo las diferencias antes y después del tratamiento (Buendía, Colás y Hernández, 1998). Por lo tanto, para complementar la investigación y darle mayor validez, se recolectaron otros datos cualitativos y cuantitativos, relacionados con el rendimiento académico. Dichos datos se obtuvieron a través de las pruebas escritas, asignaciones y la lista de cotejo. Cabe destacar, que el promedio de notas obtenido por el grupo fue de 20.38 sobre un total de 30 puntos.

En las pruebas escritas, el promedio de notas obtenido por los alumnos y las alumnas fue de 6.10 y 3.04 para las pruebas 1 y 2 respectivamente (Tabla 3). Concretamente de 34 estudiantes, 25 aprobaron la prueba 1 y 23 la prueba 2. Con respecto a las asignaciones, el número de estudiantes aprobados fue 28, 32 y 32 para las asignaciones 1, 2 y 3 respectivamente. 
Tabla 3. Media y desviación estándar obtenidas de las pruebas y asignaciones escritas

\begin{tabular}{lccccc}
\hline $\begin{array}{c}N^{0} \text { Participantes } \\
\text { 34 }\end{array}$ & $\begin{array}{c}\text { Prueba1 } \\
\text { 10 puntos }\end{array}$ & $\begin{array}{c}\text { Prueba 2 } \\
5 \text { puntos }\end{array}$ & $\begin{array}{c}\text { Asignación1 } \\
5 \text { puntos }\end{array}$ & $\begin{array}{c}\text { Asignación2 } \\
5 \text { puntos }\end{array}$ & $\begin{array}{c}\text { Asignación3 } \\
5 \text { puntos }\end{array}$ \\
\hline Media & 6.10 & 3.04 & 3.02 & 3.91 & 4.29 \\
Desviación Típica & 2.18 & 1.10 & 1.09 & 0.86 & 0.94 \\
\hline
\end{tabular}

Se observa en los resultados, de la Tabla 3, que los alumnos y las alumnas mejoraron su promedio de notas a medida que fueron elaborando las asignaciones, estando los datos menos dispersos y concentrados alrededor de la media en las últimas asignaciones.

Por otro lado, los registros en las listas de cotejo de las conductas de los alumnos y las alumnas observadas en las pruebas y asignaciones mediante listas de cotejo, arrojaron un alto porcentaje en las conductas relativas al conocimiento de símbolos, algoritmos, conceptos, teoremas y leyes, representaciones gráficas, estrategias y técnicas por emplear, tal como se muestra en la Tabla 4.

Tabla 4. Registro de observaciones realizado a las asignaciones y pruebas escritas

\begin{tabular}{|c|c|c|c|c|c|c|}
\hline \multirow{2}{*}{$\begin{array}{c}\text { Variable } \\
\text { Conocimientos }\end{array}$} & \multirow[b]{2}{*}{ Aspectos observados } & \multicolumn{5}{|c|}{$\%$ de Conductas Observadas } \\
\hline & & A1 & A2 & A3 & P1 & P2 \\
\hline \multirow[t]{6}{*}{ Conceptual } & Conoce los símbolos utilizados & 91.2 & 91.2 & 97.1 & 97.1 & 97.1 \\
\hline & Conoce los algoritmos & 85.3 & 88.2 & 97.1 & 88.2 & 85.3 \\
\hline & Conoce los conceptos & 82.4 & 88.2 & 94.1 & 88.2 & 85.3 \\
\hline & $\begin{array}{l}\text { Tiene conocimiento de los teoremas y } \\
\text { leyes }\end{array}$ & 82.4 & 85.3 & 88.2 & 94.1 & 55.9 \\
\hline & Conoce las representaciones gráficas & 64.7 & 79.4 & 94.1 & 64.7 & 85.3 \\
\hline & $\begin{array}{l}\text { Conoce las estrategias y técnicas a } \\
\text { emplear }\end{array}$ & 55.9 & 76.5 & 85.3 & 88.2 & 97.1 \\
\hline \multirow[t]{9}{*}{ Procedimientos } & Maneja los símbolos utilizados & 85.3 & 85.3 & 94.1 & 100.0 & 94.1 \\
\hline & $\begin{array}{l}\text { Aplica las reglas o algoritmos de } \\
\text { manera correcta }\end{array}$ & 50.0 & 70.6 & 85.3 & 50.0 & 44.1 \\
\hline & $\begin{array}{l}\text { Aplica los conceptos de manera } \\
\text { apropiada }\end{array}$ & 41.2 & 61.8 & 64.7 & 41.2 & 32.4 \\
\hline & $\begin{array}{l}\text { Aplica los teoremas y leyes de forma } \\
\text { lógica }\end{array}$ & 35.3 & 52.9 & 61.8 & 35.3 & 38.2 \\
\hline & Realiza las representaciones gráficas & 52.9 & 64.7 & 91.2 & 55.9 & 58.8 \\
\hline & $\begin{array}{l}\text { Maneja las técnicas utilizadas en la } \\
\text { resolución de ejercicios }\end{array}$ & 35.3 & 58.8 & 79.4 & 32.4 & 38.2 \\
\hline & $\begin{array}{l}\text { Explica la técnica o estrategia } \\
\text { empleada }\end{array}$ & 23.5 & 47.4 & 70.6 & 23.5 & 23.5 \\
\hline & Usa diversas estrategias o técnicas & 8.8 & 8.8 & 14.7 & 17.6 & 8.8 \\
\hline & $\begin{array}{l}\text { Presenta un trabajo en forma lógica y } \\
\text { ordenada }\end{array}$ & 79.4 & 91.2 & 97.1 & 97.1 & 99.1 \\
\hline
\end{tabular}


En relación con los conocimientos de procedimientos (ver Tabla 4), se observó que los alumnos y las alumnas presentaron mayor porcentaje de conductas en manejo de los símbolos, aplicación de reglas o algoritmos, aplicación de los conceptos de manera apropiada, realización de representaciones gráficas, manejo de técnicas utilizadas en la resolución de ejercicios y presentación del trabajo en forma lógica y ordenada. Sin embargo, las habilidades relacionadas con: explicar las estrategias empleadas, usar diversas estrategias o técnicas, aplicar los teoremas y leyes de manera apropiada y lógica, se observaron en menor porcentaje.

Sobre la base de lo expuesto, con estos resultados significativos se puede rechazar la hipótesis nula y establecer la posibilidad de que existe relación entre las estrategias instruccionales basadas en el software matemático y la obtención de mejoras del conocimiento de la asignatura en la población de estudiantes.

\subsection{Segunda hipótesis de investigación}

Para verificar la hipótesis, se llevó a cabo un registro de observaciones sobre los procedimientos empleados por los discentes. En esta actividad, los investigadores e investigadoras llevaron a cabo el registro en lista de cotejo, calculándose luego los porcentajes simples de acuerdos e indicando en líneas generales consistencia en las observaciones.

En cuanto al porcentaje del registro de las conductas observadas en horas de consulta (ver Tabla 5), se observó un aumento progresivo en los participantes al ejecutar procedimientos. Sin embargo, se evidencia en menor porcentaje el empleo de habilidades relacionadas con: (a) aplicar conceptos a situaciones nuevas; (b) modelar, explicar y aplicar una estrategia; (c) generar nuevos procedimientos y (d) realizar generalizaciones. Así mismo, se observó en mayor porcentaje el empleo de las siguientes habilidades: (a) usa contradicciones para aclarar conceptos, (b) realización de procedimientos de ensayo y error, (c) realización de inferencias, (d) toma de decisiones de acuerdo con criterios válidos, (e) evaluación de la veracidad de los resultados con base en procedimientos válidos, (f) proponer y explorar conexiones entre conceptos y (g) comunicación de los resultados en forma clara y lógica. 
Tabla 5. Registro de observaciones realizado en las horas de consulta

\begin{tabular}{|c|c|c|c|}
\hline \multirow[t]{2}{*}{ Aspectos Observados } & \multicolumn{3}{|c|}{$\%$ conductas observadas } \\
\hline & A1 & A2 & A3 \\
\hline Usa contradicciones para aclarar conceptos & 77.2 & 85.5 & 88.6 \\
\hline Realiza procedimientos de ensayo y error & 77.2 & 82.9 & 88.6 \\
\hline Propone y explora conexiones entre conceptos & 40.0 & 51.5 & 57.2 \\
\hline Aplica los conceptos a situaciones nuevas & 37.2 & 42.9 & 48.6 \\
\hline Toma decisiones de acuerdo con criterios válidos & 37.2 & 54.3 & 65.8 \\
\hline Modela, explica y aplica una estrategia & 17.2 & 31.5 & 37.2 \\
\hline $\begin{array}{l}\text { Evalúa la veracidad de los resultados, con base en } \\
\text { procedimientos válidos }\end{array}$ & 34.3 & 48.6 & 60.0 \\
\hline Genera nuevos procedimientos & 14.3 & 17.2 & 20.0 \\
\hline Realiza inferencias & 31.5 & 45.8 & 51.5 \\
\hline Realiza generalizaciones & 14.3 & 20.0 & 25.8 \\
\hline Comunica resultados en forma clara y lógica & 54.3 & 65.8 & 77.2 \\
\hline
\end{tabular}

Nota. A1 indica la asignación 1, A2 indica la asignación 2 y A3 indica la asignación 3

Para enriquecer las observaciones registradas a través de las listas de cotejo, se realizó una entrevista semiestructurada a las personas participantes del estudio. Las respuestas se transcribieron y se agruparon según los indicadores de la variable procedimientos. Algunas de estas respuestas se muestran en el Apéndice. En la Tabla 6 se observa un resumen de los resultados.

En relación con el indicador usa contradicciones para aclarar conceptos (ver Apéndice, Tabla A1), las opiniones fueron favorables en torno al empleo del software para realizar: (a) ensayos a través de ejemplos, (b) cálculos complicados de manera rápida y confiable, (c) secuencias donde se visualizan conceptos y (d) gráficas complejas en forma precisa y rápida. Así mismo, las opiniones también reflejaron el uso del software como apoyo para establecer a través de ejemplos concretos: contradicciones, visualizaciones de gráficas, verificaciones de suposiciones, relaciones entre conceptos, así como relaciones y contradicciones entre gráficas y cálculos. Por lo tanto, el software sirvió al discente como soporte de ideas y resultados, y le brindó la posibilidad de reflexionar sobre estos para analizar respuestas, experimentar y observar contradicciones. Todo esto, con el objetivo de aclarar dudas y facilitar la comprensión de los conceptos abstractos. En tal sentido, fue un soporte de sus planteamientos, ayudándole en su proceso de visualización e integración de ideas. No obstante, dichas opiniones reflejaron que este proceso requiere de la revisión constante de la teoría, ver si dicha herramienta es aplicable al contenido a aprender y conocer bien la sintaxis del programa. 
Tabla 6. Resultados Obtenidos en la Entrevista Semi-Estructurada

\begin{tabular}{lccc}
\hline & \multicolumn{3}{c}{ Opinión } \\
\cline { 2 - 4 }$\quad$ Indicador & Favorable & Desfavorable & Otro \\
Usa contradicciones para aclarar conceptos & 17 & 1 & 1 \\
Realiza procedimientos de ensayo y error & 16 & 1 & 4 \\
Propone y explora conexiones entre conceptos & 18 & 1 & 0 \\
Aplica el concepto a situaciones nuevas & 17 & 3 & 0 \\
Toma decisiones de acuerdo con criterios válidos & 15 & 0 & 2 \\
Modelar, explica y aplica una estrategia & 14 & 1 & 2 \\
Evalúa la veracidad de los resultados, con base en & 12 & 2 & 1 \\
procedimientos válidos & & & 0 \\
Genera nuevos procedimientos & 15 & 1 & 2 \\
Realiza inferencias & 16 & 1 & 0 \\
Realiza generalizaciones & 14 & 1 & \\
Comunica resultados en forma clara y lógica & 14 & 3 & \\
\hline
\end{tabular}

Relacionado con el indicador referente a la realización de procedimientos de ensayo y error (ver Apéndice, Tabla A2), los estudiantes y las estudiantes consideraron que se apoyaron en el software para ejecutar sondeos, variar las condiciones y parámetros del ejercicio (aumentar el número de rectángulos en las sumas de Riemann), programar secuencias con el objetivo de explorar y comprender conceptos, realizar cálculos de manera rápida y confiable, variar gráficas y observar los cambios ocurridos. Con base en las modificaciones y en los resultados generados por el software, realizaron análisis y comparaciones, conectando la teoría con la práctica y estableciendo conclusiones. Además, se observaron opiniones relativas al ahorro de tiempo y esfuerzo al usar el software, pues esta herramienta los ayudó a realizar cálculos y gráficas de manera rápida y precisa. Es evidente, que con las estrategias el discente pudo experimentar, observar, analizar y establecer conclusiones. Por lo tanto, de alguna manera ejecutó procedimientos de ensayo y error para realizar análisis.

Para el indicador propone y explora conexiones entre conceptos (ver Apéndice, Tabla A3), las opiniones mostraron que los alumnos y las alumnas se apoyaron en las capacidades informáticas del software. La rapidez y precisión al realizar los cálculos los ayudó a ensayar procedimientos, explorar conceptos, establecer relaciones, así como aclarar dudas. Además, pudieron observar y explorar conexiones entre los conceptos de límite, integral definida, sumas de Riemann, área, entre otros. Sin embargo, las opiniones indicaron que tuvieron que indagar conocimientos teóricos para entender mejor lo que estaban ejecutando y establecer conclusiones. Así mismo, algunas opiniones mostraron que las capacidades informáticas del software les dieron seguridad en los cálculos y gráficas. Además, les permitieron ahorrar tiempo y esfuerzo para concentrarse en entender la teoría. 
En relación con el indicador aplica el concepto a situaciones nuevas (ver Apéndice, Tabla A4), las opiniones se orientaron hacia el empleo del software como apoyo al alumnado para organizar mejor los conocimientos, programar secuencias, observar la construcción de conceptos, variar las situaciones, analizar diversas pruebas de ensayo y error. Es decir, tuvieron la oportunidad de balancear mejor la teoría con la práctica, a pesar de que las opiniones también arrojaron la poca utilidad del software para aprender algoritmos matemáticos. De igual manera, las opiniones apuntaron a que el proceso de análisis y reflexión no fue fácil pues se debe pensar más en los conocimientos teóricos. Sin embargo, algunas opiniones señalaron que al usar el software la asesoría del docente es fundamental (ver Tabla 7).

Tabla 7. Algunas opiniones de los estudiantes.

\begin{tabular}{l}
\hline Opiniones \\
\hline “...pensamos que el apoyo y dirección del profesor es importante..." \\
"El software nos ayudó a realizar cálculos y gráficas, así como en su facilidad para organizar la \\
información. Nosotros debimos planificar, tomar decisiones e incluso aclarar dudas. Claro bajo la \\
asesoría del profesor, la cual es necesaria" \\
"...creemos, que para implementar el software para aprender matemática la orientación del \\
profesor es primordial..." \\
“...en muchas ocasiones debimos producir ideas, expresarlas correctamente, tomando como \\
soporte los cálculos y gráficas, desarrollar las mismas considerando la teoría. Aquí la orientación \\
del profesor es básica" \\
"... tuvimos que organizar muy bien el trabajo, tanto los aspectos teóricos como prácticos. El \\
software es muy útil ya que pudimos escribir teoría, hacer cálculos, gráficas y tablas, sin embargo \\
siempre es bueno contar con la asesoría del docente". \\
"El software nos ayudó mucho con su rapidez y exactitud. Sin embargo, nosotros debimos a \\
organizar nuestras ideas para generar conclusiones. Esto nos dio ideas para generar \\
procedimientos y verificarlos. Creemos que el apoyo del profesor es esencial" \\
\hline
\end{tabular}

Para el indicador relacionado con la toma de decisiones de acuerdo con criterios válidos (ver Apéndice, Tabla A5), las opiniones nuevamente arrojaron el hecho de aprovechar las capacidades gráficas y de cálculo del software, pues esta herramienta les permitió calcular y graficar de manera rápida y precisa, realizar ensayos y cotejar resultados, hacer alejamientos y acercamientos a las gráficas, chequear procedimientos gráficos y analíticos. Sin embargo, el/la estudiante debió reflexionar sobre los resultados, analizarlos, cotejarlos con la teoría y establecer conclusiones. Por lo tanto, las opiniones confirmaron que el software sirvió de herramienta de apoyo para ver detalles y tomar mejores decisiones.

Sobre la utilidad de software para modelar, explicar y aplicar una estrategia (ver Apéndice, Tabla A6), según las opiniones de los alumnos y de las alumnas, parece que conocer la estructura y sintaxis del software es importante, así como tener buen dominio teórico del contenido por estudiar para poder modelar, explicar y aplicar una estrategia. De 
nuevo, las opiniones se orientaron hacia el uso de las capacidades informáticas del software para realizar ensayos de ideas, organizar datos y ensayar procedimientos analíticos. Todo esto, brindó la oportunidad de experimentar, reflexionar y diseñar estrategias.

Para las opiniones relacionadas con el uso del software como herramienta de apoyo para evaluar la veracidad de los resultados sobre la base de criterios válidos (ver Apéndice, Tabla A7), las opiniones apuntaron hacia la idea de aprovechar la rapidez y exactitud de los cálculos del software, tanto para ahorrar tiempo en verificar los resultados, como para programar secuencias que permitan observar el proceso de construcción de un concepto. Es decir, poder revisar, ajustar o corregir el proceso según sea necesario. También, señalaron que es una herramienta útil para comprobar respuestas de ejercicios realizados en forma manual (gráficas, cálculos de integrales). Este chequeo les ayudó a verificar si los procedimientos y técnicas usados fueron correctos, o si tenían que revisarlos. Sin embargo, los alumnos y las alumnas estuvieron conscientes de que existen situaciones en las que no se puede usar el software. Por ejemplo, para aprender procedimientos de graficación.

Con respecto a las opiniones del grupo de participantes sobre el uso del software como apoyo para generar nuevos procedimientos (ver Apéndice, Tabla A8), nuevamente los alumnos y las alumnas opinaron que el apoyo está en las capacidades informáticas del software, pues dichas capacidades, ayudaron al discente a disponer de más tiempo para pensar en la resolución del ejercicio, estimulando su creatividad e iniciativa. Si embargo, las opiniones también apuntaron hacia la importancia del proceso de revisar tanto la teoría como el proceso seguido para generar procedimientos. Dicho proceso de revisión les sirvió para idear procedimientos que les permitieron realizar mejores análisis, organizar las ideas y tomar decisiones.

Sobre el software como herramienta de apoyo para hacer inferencias (ver Apéndice, Tabla A9), opinaron que pudieron resolver ejercicios concretos y relacionarlos entre sí, así como realizar cálculos precisos y rápidos. Además, indicaron que al usar el software los resultados siempre se tienen a mano en la hoja de trabajo, por lo que pudieron ser consultados cuando era necesario, o calcular otros si hacen falta. Por lo tanto, al reflexionar y analizar sobre estos resultados, se pueden generar ideas para hacer algunas inferencias. Aunque según los alumnos y alumnas este proceso no es fácil.

En correspondencia con la utilidad de software como herramienta de apoyo para realizar generalizaciones matemáticas (ver Apéndice, Tabla A10), las respuestas apuntan hacia la utilización de las capacidades informáticas del software como apoyo para generar procedimientos, que permitan entender mejor las generalizaciones matemáticas. Según el 
grupo de participantes, el software fue usado para ejecutar exploraciones de conceptos y teoremas a través de ejercicios concretos, hacer comparaciones entre los resultados para ampliar ideas sobre un concepto, realizar verificaciones, reflexionar y comparar los resultados con la teoría. Sin embargo, los estudiantes y las estudiantes afirmaron que para concretar generalizaciones hay que estudiar constantemente los resultados y reflexionar sobre ellos, señalando que este proceso no es fácil. Por lo tanto, las opiniones apuntaron hacia el software como herramienta de apoyo para entender generalizaciones matemáticas.

En relación con a la opinión sobre el empleo del software como herramienta de apoyo para comunicar los resultados en forma clara y lógica (ver Apéndice, Tabla A11). Las opiniones fueron favorables hacia el aprovechamiento de las capacidades del software para organizar la teoría y realizar cálculos y gráficas, además de diseñar secuencias e introducir explicaciones en texto para expresar mejor lo que querían comunicar. Sin embargo, manifestaron la presencia de inconvenientes como manejo correcto de la sintaxis del software.

Sobre la base de lo expuesto, se establece la posibilidad de que existe relación entre las estrategias instruccionales basadas en el software matemático y el desarrollo de habilidades relacionadas con el uso de procedimientos en la población de estudiantes. Sin embargo, se sugiere realizar un estudio más profundo en torno a ello, pues los alumnos y las alumnas solo pusieron en práctica las habilidades cognoscitivas y metacognitivas en un tema de la asignatura Matemática II. Para determinar un verdadero impacto, es necesario extender la cobertura del software a toda la asignatura y a otros grupos de alumnos. De esta manera, los efectos de la intervención educativa pueden corroborarse con resultados de cursos anteriores. Así mismo, se hace necesario realizar un seguimiento a los dicentes que participaron en el estudio para verificar si siguen usando las habilidades en cursos superiores (Matemática III) y perfeccionando las mismas.

\section{Discusión}

Los resultados mostraron que los conocimientos del alumnado mejoraron con la aplicación de las estrategias basadas en el software matemático, pues su rendimiento académico en líneas generales fue bueno. En cuanto a los conocimientos conceptuales se evidenció que la población estudiantil conocía los símbolos, los algoritmos, los conceptos, los teoremas, las representaciones gráficas, las estrategias y las técnicas por emplear. En cuanto a los conocimientos de procedimientos, se observó en mayor porcentaje la presencia de conductas como manejo de símbolos, realización de representaciones gráficas, la 
aplicación de conceptos y algoritmos, presentación del trabajo en forma lógica y ordenada. Además, en menor porcentaje se observó la aplicación de teoremas en forma lógica, manejo de técnicas en la resolución de ejercicios, explicación y uso de diversas técnicas. No obstante, se observó mayor porcentaje de conductas en las últimas asignaciones y pruebas escritas. Porcentaje que refleja la aparición de la conducta y que puede indicar la posibilidad de una mejora en los conocimientos.

Lo expuesto denota que es posible integrar el software matemático al trabajo intelectual del alumnado, tal como lo señalaron Oteiza y Silva (2001), enfocando el uso del software hacia la enseñanza de la asignatura y facilitando que el discente aprenda a procesar la información de la materia. Estos resultados fueron señalados por Esteban (2002), quien manifestó la idea de usar las tecnologías como herramientas para promover el conocimiento en los alumnos y en las alumnas, y dar apoyo para la comprensión de conceptos.

Por otra parte, se evidenció que las estrategias empleadas, ayudaron a los discentes a comparar, visualizar conceptos, verificar, graficar, programar algoritmos, conjeturar y refutar hipótesis. Por lo tanto, el software sirvió de apoyo para facilitar la comprensión y aprendizaje de los contenidos programáticos y la concretización de conceptos abstractos haciendo más fácil su asimilación. Los anteriores también son aspectos señalados por Ángel y Bautista (2001), Balderas (2002), Galdo y Cociña (1998), Orellana (1999) y Queralt (2000). Así mismo, los resultados arrojaron que la población de estudiantes puso en práctica habilidades cognoscitivas y metacognitivas, pues con esta herramienta pudieron calcular, graficar, copiar, trasladar, ordenar, borrar, insertar, entre otros, lo que permitió generar y organizar las ideas para actuar posteriormente con ellas y así apoyar su proceso de aprender (Esteban, 2002).

Como se pudo notar, las opiniones dadas por los alumnos y las alumnas en las entrevistas, reflejaron que apoyándose en las capacidades informáticas del software lograron poner en práctica sus habilidades. Es decir, establecieron analogías y generalizaciones, diseñaron planes, abandonaron soluciones incorrectas e ineficientes, tomaron decisiones, regularon y organizaron estrategias e ideas, ensayaron procedimientos, supervisaron, evaluaron el proceso seguido y realizaron procedimientos de ensayo y error. Ahora bien, las opiniones indicaron que dichos procesos no son fáciles de utilizar pues requieren mayor esfuerzo y dedicación. También se puede inferir el uso de habilidades como formulación, planificación, ejecución, supervisión de acciones y toma de decisiones relacionadas al cuándo, cómo y dónde utilizar el computador. Cabe destacar que todas estas habilidades pertenecen al pensamiento complejo y, según Martínez (1999), son aspectos esenciales para 
el desarrollo de las habilidades metacognitivas. Además, poner en práctica habilidades cognoscitivas y metacognitivas es importante, pues estas pueden ser desarrolladas a través de su ejercitación constante (Cuicas, 1997; Sánchez, 2002). Por lo tanto, se fortalece la idea de que las herramientas informáticas, vinculándolas a experiencias significativas, se constituyen en herramientas cognitivas que los alumnos y las alumnas pueden usar para desarrollar habilidades del pensamiento (Jonassen, Carr y Ping, 1998).

Sobre la base de lo expuesto, se refuerzan las siguientes ideas detectadas en la revisión de la literatura: (a) el uso de tecnologías en la enseñanza de la matemática permiten en el discente el desarrollo de habilidades del pensamiento como: explorar, inferir, hacer conjeturas, justificar, argumentar y de esta forma construir su propio conocimiento (Fernández, Izquierdo y Lima, 2000); (b) el computador puede constituir herramientas cognitivas que el discente logra utilizar para estimular y desarrollar habilidades del pensamiento (Jonassen, Carr y Ping, 1998) y (c) el computador le permite aplicar eficientemente sus esfuerzos y poner en marcha mecanismos más complejos del pensamiento (Marqués, 2001).

Lo expuesto, evidencia que el empleo de estrategias instruccionales facilitó que el/la estudiante pusiera en práctica estrategias cognitivas de orden superior y otras destrezas intelectuales (Ríos, 1998). No obstante, en opinión de los investigadores e investigadoras se debe seguir indagando sobre este aspecto, guiando la investigación hacia la búsqueda de caminos efectivos para la adquisición y mejora permanente de estas destrezas.

\section{Conclusiones}

Los estudiantes y las estudiantes que participaron en la experiencia la consideraron beneficiosa para el proceso de enseñanza-aprendizaje de la matemática. Así mismo, el uso del software permitió al discente realizar ensayos, experimentos, demostraciones y reflexión. Le facilitó visualizar el sentido que para él tiene ese nuevo aprendizaje al relacionarlo con sus conocimientos previos, además, permitió dar la oportunidad al discente para plantear hipótesis de manera individual o en grupo (justificando su planteamiento), para concluir con la aceptación o modificación de su hipótesis, lo que provoca cambios significativos en el ambiente de aula, con clases más dinámicas, participativas y centradas en el estudiante. Además, se evidenció que con el empleo de estas estrategias se conformó un ambiente de aprendizaje que invitó a la reflexión, al análisis, a la actitud crítica en la solución de problemas y a la toma de decisiones. Sirviendo la herramienta informática utilizada como elemento de motivación. En tal sentido, la realización de esta investigación ha permitido 
enriquecer el conocimiento sobre el uso del software matemático como herramienta cognitiva, para mejorar la comprensión y el aprendizaje del tema de la integral definida en los estudiantes y las estudiantes.

Con la aplicación de las estrategias los hallazgos de la investigación fueron: (a) los conocimientos de la población estudiantil mejoraron; y (b) la población de estudiantes pusieron en práctica sus procedimientos, es decir, sus habilidades cognoscitivas y metacognitivas. Por lo tanto el estudio aportó evidencias para utilizar el software matemático bajo una metodología instruccional constructivista. No obstante, sería conveniente realizar otras pruebas ya que estos resultados son válidos para este grupo de discentes en particular.

\section{Referencias}

Ángel, Juan, y Bautista, Guillermo. (2001). Didácticas de las matemáticas en enseñanza superior: La utilización de software especializado. Recuperado el 12 de Enero de 2005, de http:// www.uoc.edu/web/esp/art/uoc/0107030/mates.html

Anido, Mercedes, López, Roberto, y Rubio, Héctor. (2006). Las supersuperficies en el aprendizaje de la geometría. Recuperado el 20 de Diciembre de 2006, de http://redalyc.uaemex.mx/redalyc/pdf/335/33590302.pdf

Balderas, Angel. (2002). Didácticas de las matemáticas en Internet. Comunidades educativas y ambientes virtuales: Situación actual y perspectiva. Recuperado el 21 de Enero de 2005, de http://informaticaeducativa.com/coloquios/mesas/tres/angel/didactica.html

Bavaresco, Aura. (2001). Proceso metodológico de la investigación: Cómo hacer un diseño de investigación. Maracaibo, Venezuela: Universidad del Zulia.

Buendía, Leonor, Colás, Pilar, y Hernández, Fuensanta. (1998). Métodos de investigación en psicopedagogía. Madrid, España: McGraw Hill-Interamericana.

Cataldi, Zulma. (2000). Metodología de diseño, desarrollo y evaluación de software educativo. Recuperado el 8 de Agosto de 2005, de http://www.fi.uba.ar/laboratorios/lsi/cataldi-tesisdemagistereninformatica.pdf

Coll, Cesar, Pozo, Juan, Sarabia, Bernabé, y Valls, Enric. (1992), Los contenidos de la reforma: Enseñanza y aprendizaje de conceptos, procedimientos y actividades. Madrid, España: Santillana/Aula XXI.

Cuicas, Marisol. (1997). Procesos metacognitivos desarrollados por los alumnos cuando resuelven problemas matemáticos. Tesis de maestría no publicada, UCLA-UNEXPO-UPEL, Barquisimeto, Lara, Venezuela. 
Dede, Chips. (2000). Aprendiendo con tecnología. Buenos Aires, Argentina: Paidós Ibérica, S. A.

Dávila, Nancy, Hernández, Juan, García, Dolores, Martel, María, Gómez, Emilio, y Vásquez, Francisco. (1998). El uso del ordenador en las matemáticas para la economía y la empresa: Una experiencia en la Universidad de las Palmas de G.C. Recuperado el 21 de Enero 2005, de http://www.uv.es/asepuma/jornadas/santiago/29.PDF.

De Guzmán, Miguel. (1993). Tendencias innovadoras en educación matemática. Recuperado el 10 de Febrero de 2005, de http://www.mat.ucm.es/deptos/am/guzman tendencia/ensen.htm

Díaz, Frida, y Hernández, Gerardo. (2002). Estrategias docentes para un aprendizaje significativo: Una interpretación constructivista (2a ed.). México, D.F.: McGrawHill Interamericana.

Esteban, Manuel. (2002). El diseño de entornos de aprendizaje constructivista. Recuperado el 1 de Agosto de 2005, de http://www.um.es/ead/red/6/documento6.pdf

Fallad, Jalil. (1999). Software educativo para la asignatura de matemática I de nivel de licenciatura. Recuperado el 2 de Junio, 2005, de http://www.somece.org.mx/memorias/1999/inditema.htm

Fernández, Francisco, Izquierdo, José, y Lima, Sylvia. (2000). Experiencias en la estructuración de clases de matemáticas empleando asistentes matemáticos y colección de tutoriales hipermediales. Recuperado el 10 de Marzo de 2005, de http://www.c5.cl/ieinvestiga/actas/ribie2000/papers/106/

Fernández, Pita, y Díaz, Pértegas. (2003). Las ventajas e inconvenientes de los métodos cuantitativos y cualitativos, Candidus, 1, 9-10.

Flórez, Rafael. (2000). Hacia una pedagogía del conocimiento. Bogotá, Colombia: McGraw-Hill Interamericana.

Galdo, Constanza, y Cociña, Andrea. (1998). Matemática III con Mathematica en la UCA. En Cruz C., Serres Y., Bayer W., Mosquera J. y Millán O. (Eds.), Memorias III Congreso Iberoamericano de Educación Matemática (pp. 667-669). Caracas, Venezuela: FAPUVA-UCV.

Garza, Rosa., y Leventhal, Susana. (2004). Aprender cómo aprender. México, D.F.: Trillas.

Gavilán, José, Ariza, Antonio, Sánchez, Ángel, y Barroso, Ricardo. (1999). Software en el aprendizaje de las matemáticas. Recuperado el 10 de Febrero 2005, de http://www.aloj.us.es/rbarroso/Pruebas/proyectoSAM.pdf

Guedez, Maita. (2005). El aprendizaje de funciones reales con el uso de un software educativo: una experiencia didáctica con estudiantes de educación de la ULATáchira. Recuperado el 12 de Abril de 2006, de http://www.saber.ula.ve/db/ssaber/Edocs/pubelectronicas/accionpedagogica/vol14nu $\underline{\mathrm{m} 1 / \text { articulo4.pdf }}$ 
Hernández, Roberto, Fernández, Carlos, y Baptista, Pilar. (2003). Metodología de la Investigación (3a ed.). Bogotá, Colombia: McGraw-Hill Interamericana.

Hernández, Fernando, y Sancho, Juana. (1993). Para enseñar no basta con saber la asignatura. Barcelona, España: Paidós Ibérica, S. A.

Jonassen, David. (1996). Computers in the classroom: Mindtools for critical thinking. Englewood Cliffs, New Jersey: Merrill Prentice-Hall.

Jonassen, David, Carr, Chad, y Ping, Hsiu-Ping. (1998). Computers as Mindtools for Engaging Learners in Critical Thinking. Recuperado el 12 de Abril de 2005, de http://tiger.coe.missouri.edu/ jonassen/Mindtools.pdf

León, Orfelio, y Montero, Ignacio. (2003). Métodos de investigación en Psicología y educación (3a ed.). Madrid, España: McGraw-Hill Interamericana.

Marqués, Pere. (2001). Los procesos de enseñanza y aprendizaje. Recuperado el 12 de Enero de 2005, de http://dewey.uab.es/pmarques/actodid.htm

Martín, Juan. (2001). Enseñanza de procesos de pensamiento: Metodología, metacognición y transferencia. Recuperado 12 de Febrero de 2005, de http://www.uv.es/RELIEVE/v7n2/RELIEVEv7n2 2.htm

Martínez, Ana. (1999). Computadores en el aula: Herramientas mentales para la activación del pensamiento significativo. Recuperado 2 de Febrero de 2005, de http://www.revele.com.ve/pdf/agenda/vol6-n2/pag91.pdf

Meza, Adriana, y Cantarell, Lisbeth. (2002). Importancia del manejo de estrategias de aprendizaje para el uso educativo de nuevas tecnologías de información y comunicación en educación. Recuperado el 2 de Febrero de 2005, de http://funredes.org/mistica/castellano/ciberoteca/participantes/docuparti/esp doc 71.h $\underline{\mathrm{tml}}$

Orellana, Mauricio. (1999). La enseñanza del cálculo. Caracas, Venezuela: Universidad Central de Venezuela

Oteiza, Fidel, y Silva, Juan. (2001). Computadores y comunicaciones en el currículo matemático: Aplicaciones a la enseñanza secundaria. Recuperado el 10 de Mayo de 2005, de http://www.eduteka.org/pdfdir/SilvaMatematicas.pdf

Oteiza, Fidel, y Miranda, Hernán. (2001). El conocimiento matemático que se enseña en la escuela: ¿Está respondiendo a los nuevos desafíos de la educación? Recuperado el 10 de Mayo de 2005, de http://www.comenius.usach.cl/fondef/documentos.htm

Queralt, Tomás. (2000). Un enfoque constructivista en el aprendizaje de las matemáticas con las calculadoras gráficas. Recuperado el 12 de Enero de 2005, de http://www.ti.com/calc/latinoamerica/pdf/Enfoque.pdf

Ríos, Javier. (1998). El uso de la tecnología en la clase de matemáticas. Recuperado el 10 de Marzo de 2005, de http://www.niee.ufrgs.br/ribie98/TRABALHOS/126M.PDF 
Sánchez, Margarita. (2002). La investigación sobre el desarrollo y la enseñanza de las habilidades del pensamiento. Recuperado el 20 de Febrero de 2005, de http://redie.ens.uabc.mx/vol4no1/contenido-amestoy.html

Sarmiento, María. (1999). Cómo aprender a enseñar y cómo enseñar a aprender: Psicología educativa y del aprendizaje. Bogotá, Colombia: Universidad Santo Tomás.

Segura, Mayra, y Chacón, Isabel. (1996). Competitividad en la educación superior. Umbral, 11(5), 29-37.

Squires, David y McDougall, Anne. (1997). Cómo elegir y utilizar un software educativo: Guía para el profesorado. Madrid, España: Morata, S. L.

Triola, Mario. (2004). Estadística (L.E. Pineda, Trad.). México, D.F.: Pearson Addison Wesley. 


\section{Apéndice \\ Resultados de las Entrevistas Semi-Estructuradas}

Tabla A1

Respuestas a las preguntas relativas al indicador usa de contradicciones para aclarar conceptos

\footnotetext{
"Muy buena, pues te permite armar secuencias donde se visualizan los conceptos, además de utilizar gráficas para visualizarlos geométricamente"

"Es una gran ayuda, pues podemos establecer contradicciones entre conceptos para facilitar el entendimiento de otros. Por ejemplo, los conceptos de límite y de sumas de Riemann, relacionados, pueden originar el concepto de la Integral Definida"

"Consideramos muy útil intentar comprender y analizar los ejercicios usando las capacidades gráficas del software. Pues de esta forma los vemos mejor"... "Para ver contradicciones entre conceptos es excelente, te apoyas en su rapidez para realizar cálculos y gráficas confiables"

"Se pueden realizar gráficas y cálculos. Ver las relaciones y contradicciones entre ellos. Al comparar esto con la teoría, puedes entender y visualizar mejor el concepto"

“... puedes visualizar conceptos, verificar rápidamente la veracidad de algunas suposiciones y ver contradicciones... los contraejemplos son útiles para sacarte de duda"

“... nos da la posibilidad de experimentar con diversas funciones, realizar cálculos y gráficas complejas. Estos ensayos nos brindan la posibilidad de observar contradicciones, analizar respuestas y aclarar conceptos. Por lo que creemos que es un buen apoyo"

“... no fue que aumentó... con el Maple evaluamos resultados, realizamos gráficas, calculamos, observamos algunas contradicciones... creemos que ayuda bastante a aclarar dudas de conceptos y a reflexionar sobre los mismos. Es un apoyo"

"Nos permitió entender mejor la teoría,... nos ayudamos con las gráficas y cálculos realizados por el Maple para generar contradicciones y representar conceptos. Por ejemplo, nos permitió visualizar mejor el concepto de integral definida."

“... el Maple ayuda a realizar gráficas, cálculos y ver contradicciones rápidamente. Sin embargo, se tiene que analizar los mismos con base a la teoría para poder visualizar conceptos... como apoyo es buena si se aplica al contenido que se aprende"

"Nos ayudó a visualizar las matemáticas como un todo... con apoyo del software, se visualiza mejor la relación entre integrales, límites, sumas de Riemann y área. Haciendo uso de contradicciones, establecimos mejor la relación entre área e integral definida"

“... el Maple es un apoyo para realizar mejor los gráficos y cálculos. Sin embargo, para visualizar conceptos, debemos revisar la teoría para ver que está pasando en los gráficos y cálculos... se debe tratar de establecer relaciones y contradicciones entre ellos"

"Cuando realizamos sondeos con el software usando ejemplos concretos, observamos contradicciones, semejanzas y diferencias. Esto ayudó a reflexionar sobre lo que hacíamos y a examinar sus consecuencias"

“... se pueden realizar gráficas, cálculos, ver contradicciones en los cálculos que se realizan, te permite ver el concepto desde otro punto de vista... Por ejemplo, nos ayudó a aclarar el concepto de límite de una suma de Riemann"

"Hay que estudiar teoría para establecer relaciones con los resultados del Maple. Sí se le ve sentido a los conceptos abstractos. Pero debemos concentrarnos más en la teoría"

"Nos apoyamos en él para realizar ensayos y generar resultados. Esto nos permitió aclarar dudas y comprender mejor la teoría... por su rapidez en realizar cálculos y gráficas,... es buena herramienta para ensayar contradicciones y relacionar conceptos"

"Con el software pudimos ensayar diferentes situaciones y plantear contradicciones. Esto, nos permitió abandonar soluciones incorrectas o buscarle los correctivos"

"Con el Maple se realizan cálculos y gráficas complejas... concentrarnos en la teoría y el análisis de la misma. Aunque esto no fue fácil, es otra forma de ver las matemáticas"

“... con él organizamos el contenido de las asignaciones. Analizamos y pensamos en las relaciones entre la teoría y los resultados del software. Creemos, que el Maple nos ayudó a trabajar las gráficas, cálculos y teoría a la vez. Aunque no fue nada fácil realizar el análisis"
} 


\section{Tabla A2}

Respuestas a las preguntas relativas al indicador realiza procedimientos de ensayo y error

“... al revisar la teoría para justificar lo que se estaba haciendo, el análisis fue mayor. Antes aprendíamos un procedimiento y ya. Ahora es diferente, hay que conectar la teoría con la práctica... el Maple se encarga de realizar los cálculos y gráficas, nosotros variamos los parámetros y condiciones. Luego analizamos que pasó" “... con apoyo del software comparamos ecuaciones, gráficas y cálculos, además de hacer diversos sondeos. Esto último genera nuevas situaciones y la necesidad de aplicar la teoría a las mismas"

"Puedes apoyarte en el software para variar rápidamente las ecuaciones, sus cálculos y gráficas. Con el software se observa en detalle los cambios que ocurren. Esto permite establecer mejores conclusiones, realizar mejores análisis y experimentar situaciones"

"Es muy práctico para realizar procedimientos de ensayo y error. El análisis de estos resultados te permiten aclarar dudas de teoría y comparar conceptos"

"El software nos ayudó a obtener cálculos y gráficas de manera rápida. Esto es de gran utilidad. En las sumas de Riemann aumentamos el número de rectángulos, variamos la función, cambiamos el intervalo de integración, etc. El análisis de estos resultados nos permitió entender mejor los conceptos de integral definida, sumas de Riemann, área y límite"

"Con ayuda del Maple, se pueden mostrar procedimientos en detalle y evitar cálculos tediosos. Esto ayuda mucho a diseñar procedimientos de ensayo y error"

"Con el software se pueden hacer diversas pruebas y relacionar conceptos. Lo mejor del caso es que los cálculos y gráficas los hace el Maple. Esto ahorra tiempo"

"Su rapidez y precisión es una ventaja para ensayar diversos procedimientos y gráficas. Lo malo es que él no analiza, lo tenemos que hacer nosotros"

"El software te brinda la oportunidad de experimentar... los cálculos y gráficas las hace el computador. Esto es de gran ayuda para realizar procedimientos de ensayo y error"

"Aprovechamos su rapidez para generar gráficos, cálculos, así como la posibilidad de acudir a ellos cada vez...nos permitió establecer comparaciones y ensayar otras condiciones en los ejercicios"

"Con el software, pudimos pensar de manera más flexible, no nos preocupó los cálculos y las gráficas... nos permitió ensayar diferentes vías y condiciones en el ejercicio"

\section{Tabla A3}

\section{Respuestas a las preguntas relativas al indicador propone y explora conexiones entre conceptos}

"Con el software se hace la carga menos pesada, con relación a los cálculos y gráficas. Además, pudimos ver con mucha claridad la conexión entre los conceptos de límite y de la integral definida. Creemos que ayuda más a explorar conexiones que a proponer".

“... los conceptos que estudiamos con el Maple, los hicimos de manera exploratoria. Esto ayudó a relacionarlos unos con otros. Sin embargo, tuvimos que indagar la teoría para entender mejor los ejercicios, analizarlos y establecer las conclusiones"

“... pudimos entender las conexiones y relaciones entre los conceptos y teoremas... apoyándonos en él pudimos visualizar y comprender lo que significaba el límite de las sumas de Riemann"

"Pudimos observar que la matemática está conectada. Por ejemplo, el límite, la derivada, la integral. Todo se relaciona. El Maple nos ayudó ver esta conexión a través de sus procesos de graficación, cálculos y programación de secuencias"

“... descubrimos que es emocionante probar ideas en la computadora, nos sentimos seguros de los resultados. Nos brindó apoyo para estimular la imaginación y relacionar conceptos"

"En clase se establecieron relaciones entre conceptos con el Maple. Para usar el software en este sentido hay que revisar constantemente la teoría. Esto absorbe mucho tiempo"

“... pudimos relacionar conceptos como integral definida, sumas de Riemann, límite, área. Además establecimos analogías y diferencias entre los conceptos"

“... sirvió de apoyo para visualizar gráficas, manipularlas y analizarlas. Todo esto nos ayudó a establecer conexiones entre conceptos. Por ejemplo, área e integral definida"

"Las asignaciones con el software debíamos estructurarlas. Por ejemplo, el estructurar el concepto de integral definida, nos llevó a reflexionar sobre sumas de Riemann, manipular funciones, aumentar el número de rectángulos. Esto nos ayudó a explorar conexiones entre estos conceptos y a entenderlos mejor" 


\section{Tabla A4}

Respuestas a las preguntas relativas al indicador aplica los conceptos a situaciones nuevas

“... el software realizó los cálculos y las gráficas, esto es una gran ayuda... nuestro trabajo fue analizar y justificar teóricamente lo que se hizo. Creemos que entender bien la teoría te permite aplicarla mejor a diversas situaciones"

"Bueno, la relación entre estos conocimientos los hemos aprendido en las clases. Pero hay situaciones en que el Maple nos sirvió de apoyo para comprender mejor esta relación. Por ejemplo, porqué el método de Simpson es más preciso que el del trapecio"

"Cuando programamos secuencias fue de gran utilidad. Se visualiza la relación entre la teoría y a práctica. Puedes hacer diversas pruebas y variar las situaciones. Sin embargo, la guía del docente es fundamental" "En la visualización y el análisis. La teoría se entiende más fácil. Además podemos expresar de manera ordenada y clara algunos detalles de los conocimientos teóricos y relacionarlos. Aunque esto no es fácil es otra forma de ver la asignatura"

“...No es necesario desarrollar los procedimientos a mano. Esto genera tiempo para establecer relaciones entre teoría y práctica, así como realizar pruebas a otras situaciones"

"Pudimos programar secuencias y observar la construcción de conceptos. Por ejemplo de la integral definida. Verificar que dicha definición siempre es consistente si se trabaja en otras situaciones, otras funciones, etc."

"... debemos pensar en el contenido de la materia. Esto obliga a relacionar la teoría con la práctica y a diseñar situaciones para visualizar mejor el contenido y aclarar dudas"

\section{Tabla A5}

Respuestas a las preguntas relativas al indicador toma de decisiones de acuerdo con criterios válidos "Pudimos realizar cálculos y graficar de manera rápida. Dichos resultados los cotejamos con la teoría y establecimos conclusiones"

"El Maple nos ayudó a construir diferentes gráficas,... pudimos hacer alejamientos y acercamientos. Esto nos permitió ver detalles y tomar decisiones"

"Los resultados del software siempre son exactos. Por lo que pudimos hacer análisis y establecer conclusiones sin temor a equivocarnos"

"Con el software realizamos diversos ensayos y efectuamos mejores gráficas. Además los cálculos son más confiables. Todo esto nos permitió tomar mejores decisiones"

"Es práctico, puedes ensayar procedimientos y cotejar resultados. Por ejemplo, pudimos calcular la integral definida usando extremos derechos, puntos medios e izquierdos de rectángulos. Esto nos permitió chequear gráfica y analíticamente procedimientos. Verificar la exactitud de los resultados y tomar decisiones"

"Con el Maple es muy sencillo realizar ensayos cambiando la función, los intervalos, las variables. En base a los resultados... realizamos análisis y establecimos conclusiones"

"Con apoyo en el software pudimos comprender y visualizar mejor diversas interpretaciones y utilizarlas para tomar decisiones sobre la base de criterios válidos"

"'Pensamos que trabajar con el software es más difícil... hay que analizar y reflexionar sobe los resultados y tomar decisiones. Sin embargo, el software calcula y gráfica de forma rápida. Creemos que este es el apoyo, lo demás tenemos que hacerlo nosotros"

\section{Tabla A6}

Respuestas a las preguntas relativas al indicador modela, explica y aplica una estrategia

\footnotetext{
"Al principio por las indicaciones del docente. Luego, de conocer la estructura y sintaxis del software, pudimos hacer pruebas y poner en práctica la estrategia para ver si resulta"

"La sintaxis del Maple es un poco difícil,... luego de que se entiende, puedes realizar los gráficos que quieras, realizar cálculos rápidamente y apoyarte en lo que has hecho"

“... el Maple brinda oportunidad de experimentar, esto es de ayuda. Sin embargo,... la estrategia la debemos diseñar... al realizar sondeos con el Maple tienes más confianza en los resultados, esto permite diseñar estrategias o por lo menos se te ocurren ideas"

"A pesar de que el Maple muestra directamente los resultados. Uno se puede apoyar en los mismos para diseñar procedimientos. Además, se le ocurren ideas"

"El uso del software exige planificar un poco la actividad antes de enfrentarte a la tarea. Según vas obteniendo los resultados, puedes revisar el proceso y corregirlo"

"En su capacidad para realizar cálculos y gráficas, así como en su facilidad para organizar la información. Nosotros debimos planificar, tomar decisiones e incluso aclarar dudas. Claro bajo la asesoría del profesor"
} 
"El Maple nos ayudó con los cálculos y gráficas. Su rapidez en generar resultados es de ayuda,... nos queda el trabajo difícil reflexionar y discutir sobre la base de la teoría... si es un apoyo para pensar y generar algunas estrategias en base a los resultados"

\title{
Tabla A7
}

Respuestas a las preguntas relativas al indicador evalúa la veracidad de los resultados, con base en criterios válidos

\begin{abstract}
"El software es bastante exacto y rápido en sus cálculos, esto nos permitió chequear los resultados y verificar si los procedimientos y técnicas usadas eran lógicos"

“... se puede comparar constantemente los resultados. Además, en base a estos últimos se pueden conectar algunos conceptos y establecer conclusiones"

"Me da seguridad, pues con el Maple puede verificar mis respuestas. Esto me permite revisar el ejercicio, y detectar y corregir errores"

"El software te da cálculos y gráficas exactas. Pero no puedes verificar si el procedimiento o algoritmos usados. Igual te queda la duda si los ejercicios que tu hiciste, los procedimientos son válidos"

"Es una buena herramienta, ya que desarrollas ejercicios a mano y verificas los resultados con el software. Además, puedes realizar diversos ejercicios para visualizar mejor los teoremas y su interpretación gráfica" "Es útil... cuando programas secuencias, para observar la construcción de un concepto, se observa el proceso, puedes revisarlo y ajustarlo o corregirlo según sea necesario"

"El software calcula y grafica. A diferencia de una calculadora, siempre estaban disponibles en una hoja de trabajo. Por lo tanto, pudimos observar patrones de comportamiento y establecer resultados en base a estos"
\end{abstract}

\section{Tabla A8}

\section{Respuestas a las preguntas relativas al indicador genera nuevos procedimientos}

\footnotetext{
"Puedes ensayar procedimientos con mayor rapidez pues los cálculos y gráficas las hacia el software. Sin embargo, hay que analizar lo que se está haciendo y revisar la teoría para generar procedimientos" "...te despierta un poco la creatividad e iniciativa. No te da flojera inventar, los cálculos los hace el Maple"

"Siempre podíamos consultar los resultados y gráficas anteriores. Esto nos ayudó a generar algunos procedimientos, relacionados con el análisis de los datos"

"Pudimos apoyarnos en sus capacidades gráficas y de cálculo. Muchas veces no se lleva a cabo el procedimiento ideado por que los cálculos y las graficas son muy complicadas"

"En su capacidad para variar gráficas y resultados. Nuestro trabajo era reflexionar, establecer conclusiones e idear procedimientos para realizar mejores análisis. Es muy útil por su rapidez y exactitud"

"Apoyándonos en los resultados..., teníamos que reflexionar y establecer conclusiones. Este proceso no es fácil, pero te ayuda a establecer algunos procedimientos para realizar análisis y tomar decisiones"'

"El software nos ayudó mucho con su rapidez y exactitud. Sin embargo, nosotros debimos a organizar nuestras ideas para generar conclusiones. Esto nos dio ideas para generar procedimientos y verificarlos"

"Las actividades con el software nos permitieron diseñar, graficar, calcular, organizar información y analizarla. Todo esto nos estimuló en pesar en procedimientos, para detectar relaciones y explicarlas mejor"
}

\section{Tabla A9}

\section{Respuestas a las preguntas relativas al indicador realiza inferencias}

\footnotetext{
"Las inferencias las hacemos nosotros... el Maple es una herramienta de apoyo,... puedes resolver ejercicios concretos y relacionarlos entre sí. El análisis de estas situaciones puede darte ideas para hacer inferencias" “... es un buen apoyo para analizar diversas situaciones y relacionar conocimientos...se puede evaluar resultados con mayor precisión. Creemos que si logramos hacer algunas inferencias en base a lo expuesto" “... es un apoyo por que todos los resultados los tienes a la mano y puedes rápidamente calcular otros si te hacen falta. Pero se deben analizar los resultados y pensar en la teoría para realizar las inferencias" "Con el Maple se trabajan ejercicios concretos. Puedes analizar sus resultados para establecer inferencias" "Es un buen apoyo, ya que puedes resolver un ejercicio, cambiar sus parámetro, comparar los resultados cotejando con la teoría y con base lo anterior establecer algunas inferencias"

"Puedes variar gráficas y resultados de manera rápida y confiable. Reflexionar sobre ellos, nos permitió realizar algunas inferencias, aunque el proceso de análisis no es fácil"

"El software no hace inferencias, analizamos los resultados para tratar de hacerlas... es una ayuda, pues tienes todos los resultados a tu disposición en el hoja de trabajo"
} 
Tabla A10

Respuestas a las preguntas relativas al indicador realiza generalizaciones

"El Maple nos sirvió de apoyo para ampliar ideas sobre un concepto determinado,... pudimos observar la parte analítica y gráfica del mismo. Creemos que sirve más para entender las generalizaciones matemáticas" "El software es práctico y ayuda para ver la lógica de conceptos y entender mejor sus generalizaciones" "Te ayuda a entender las generalizaciones. Se pueden practicar casos aislados de las mismas" “... nos proporcionó las respuestas inmediatas. No se observa el procedimiento. Sin embargo, en base a los resultados se pueden entender algunas generalizaciones..."

"...se pueden afianzar los contenidos discutidos en clase. Se entienden mejor los conceptos y teoremas"

"Puedes experimentar con ejercicios específicos. Sin embargo, sobre los resultados puedes verificar la teoría y entender mejor las generalizaciones"

"Es muy útil e interactivo, puedes variar gráficas y resultados. Compararlos en base a la teoría. Esto permite entender mejor las generalizaciones matemáticas y aplicarlas mejor"

“... es una buena herramienta para estimular el pensamiento... para hacer generalizaciones hay que estudiar constantemente los resultados y reflexionar sobre ellos. Creemos que el apoyo está en su rapidez y exactitud"

Tabla A11

Respuestas a las preguntas relativas al indicador comunica resultados en forma clara y lógica

“...el software nos permitió organizar la teoría, y realizar los cálculos y gráficas al mismo tiempo. Esto es una ventaja para comunicar mejor los resultados. Lo malo es la sintaxis del software, es un poco complicada"

“... nos permitió organizar mejor las ideas, explicarlas mejor pues podíamos realizar gráficos, cálculos, y apoyarnos en ellos para expresar mejor lo que queríamos decir".

"Si, nos permitió escribir texto, realizar cálculos, programar secuencias, graficar. Todo esto en forma lógica y ordenada según nuestra conveniencia"

"Si, pudimos organizar mejor la información y paralelo a ello, hacer gráficas y cálculos precisos. Esto nos ayudó mucho a comunicar los resultados y a establecer conclusiones"

"Sus capacidades gráficas y de calculo, nos permitió generar mejores resultados. Además, pudimos agregar explicaciones. Con él comunica mejor los resultados, siempre y cuando el trabajo esté bien organizado"

"Si, pudimos realizar gráficas, cálculos, tablas, programar secuencias. Además, escribir comentarios sobre los mismos. Creo que nos obligó a ser más organizados a la hora de comunicar resultados"

"...sí, en muchas ocasiones debimos producir ideas, expresarlas correctamente, tomando como soporte los cálculos y gráficas, desarrollar las mismas considerando la teoría y finalmente revisarlas para ver si no habían incoherencias, errores ortográficos..." 